\title{
Erratum to: Lentiviral-Mediated Netrin-1 Overexpression Improves Motor and Sensory Functions in SCT Rats Associated with SYP and GAP-43 Expressions
}

\author{
Xue Fei Han ${ }^{1} \cdot$ Yuan Zhang ${ }^{1,3} \cdot$ Liu Lin Xiong ${ }^{2} \cdot$ Yang $\mathrm{Xu}^{2} \cdot$ Piao Zhang ${ }^{1} \cdot$ \\ Qing Jie $\mathrm{Xia}^{2}$ - Ting Hua Wang ${ }^{1,2} \cdot$ Ying Chun $\mathrm{Ba}^{1}$
}

Published online: 14 March 2016

(C) Springer Science+Business Media New York 2016

\section{Erratum to: Mol Neurobiol}

DOI 10.1007/s12035-016-9723-7

The given names of the authors in the online published article have corrections. The authors do hereby correct these as Xue Fei Han, Yuan Zhang, Liu Lin Xiong, Yang Xu, Piao Zhang, Qing Jie Xia, Ting Hua Wang, Ying Chun Ba.

Moreover, they want to add an article note regarding the primary corresponding author and to add another affiliation for Yuan Zhang. These are presented below.

Xue Fei Han, Yuan Zhang, Liu Lin Xiong, Qing Jie Xia, Ting Hua Wang and Ying Chun Ba contributed equally to this work.

Ying Chun $\mathrm{Ba}$ is the first corresponding author.

The online version of the original article can be found at http://dx.doi.org/ 10.1007/s12035-016-9723-7.

Qing Jie Xia

xiaqj2005@126.com

$\triangle$ Ting Hua Wang

tinghua_neuron@263.net

$\triangle$ Ying Chun $\mathrm{Ba}$

byc71206@163.com

1 Institute of Neuroscience and Department of Anatomy, Kunming Medical University, Kunming 650000, China

2 Department of Anesthesia, Institute of Neurological Disease, Translational Neuroscience Center, West China Hospital, Sichuan University, Chengdu 610041, People's Republic of China

3 Intensive Care Unit, Pujiang People's Hospital, Chengdu City, Sichuan Province, China 